\title{
IdeaCrepe: Creativity Support Tool With History Layers
}

\author{
Nagayoshi NAKAZONO, Kazuo MISUE, and Jiro TANAKA \\ Department of Computer Science, \\ Graduate School of Systems and Information Engineering, University of Tsukuba \\ 1-1-1 Tennoudai, Tsukuba, Ibaraki, 305-8573, Japan \\ \{zono, misue, jiro\}@iplab.cs.tsukuba.ac.jp
}

\begin{abstract}
We developed a creativity support tool called IdeaCrepe that manages the history of ideas using a layered structure. Handling ideas with a creativity support tool that is able to record history is very useful for understanding these ideas, the evolution of the ideas over time, and the relationships between the ideas. Looking back through the history of creative activity may contribute to the further development of ideas. In this research, we introduce a concept of "layered structure" based on a time series to manage the history. Using this structure, we can look back at past states of ideas, and examine their development. These features can manage the creation and modification of ideas.
\end{abstract}

\section{Introduction}

We developed a creativity support tool called "IdeaCrepe" that manages the history of ideas using a layered structure. This tool has a history management function with which we can see the evolution of states of ideas easily. Recalling when ideas were created or what the previous states were is difficult, because successive ideas are created intermittently and frequently. Moreover, generally, we cannot check previous states when we want to because they are overwritten by more recent developments.

We developed a creativity support tool that enables us to look back at the history of our creative process. We think intuitively that recalling previous trends, failures, and successes will make our future activities better. We introduce a recall function and then examine the tool.

\section{History Management in Creativity Support Tool}

\subsection{Methods for Creative Activities}

There are currently any propositions of methods that can support our creative activities. Some of those methods use network diagrams which are composed of nodes (ideas) and edges (relationship lines). We selected a network diagram method for our creativity support tool. 


\subsection{Looking Back Makes New Ideas}

During many creative activities, we frequently compile observations about one theme. In activities developed in a time series, one important factor in deciding the guideline of future activities is looking back at the history of the creative process. One way of doing this is by checking previous states when ideas were created.

The general method of saving work is by overwriting it. The computer always saves the most recent snapshots of data. If you want to check previous state, you must save each snapshot without overwriting the data, and then compare multiple snapshots.

This problem is also suited to creativity support tools. We developed a creativity support tool called IdeaCrepe that implements a framework of data processing to manage the history of the creative process. We focused on a creativity support tool that uses a network diagram.

\section{$3 \quad$ Layered History Structure}

We employ a layered structure [7] as a method to manage the history of creative activity. Network data of creativity is managed separately as several layers, not as a single diagram, and each one depending on the update time. We display the data and color it in a flexible manner. This approach helps users to read large amounts of information from network diagrams.

\subsection{History Management of Network Using Layered Structure}

In IdeaCrepe, ideas produced through a creative activity are expressed as a network diagram using a concept of layered structure as shown in Fig. 1. The structure is similar to layers of transparencies like those used which an overhead projector. Changes from each update are saved on each layer.

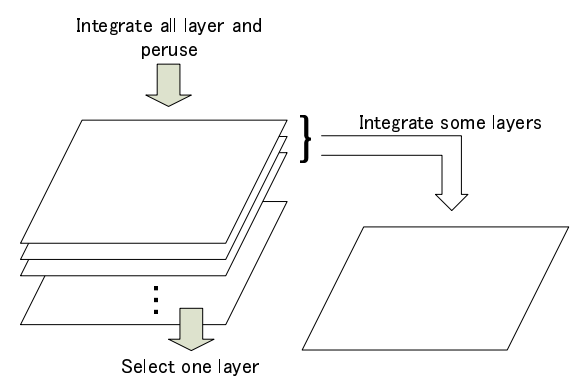

Fig. 1. Conceptual image of layered structure 


\subsection{Application to Creativity Support}

Our concept is that each layer has only one event. When an event is made, a new layer is added. The operations piling layers represent the development of knowledge during creative activity. Due to this method, the order in which events were created is clear, so we can manage the history of the creative activities more certainly. As the history is recorded in layers, we call this method a "layered history structure."

In a layered history structure, events are organized according to a time series and users can look at the optional layers easily. This merit causes that users can see the order in which their ideas created. With a layered history structure, users can grasp how much their ideas have created over a period of time, unlike existing methods that save snapshots. With the layered history structure, users can see the evolution of the creative process in more detail than is possible with existing methods.

\section{IdeaCrepe - Creativity Support Tool}

We developed a knowledge creativity support tool called "IdeaCrepe" that has a layered history structure. This tool manages ideas by using network diagrams in the same way as some existing tools.

\subsection{Operations with Tool}

Users perform various operations using IdeaCrepe. The tool provides the following operations:

- Create node: creates a node for each individual idea

- Create edge: creates an edge (a relationship line) to link ideas

- Change label: changes the label of a node or an edge

- Move node: moves the position of a node

- delete node: deletes a node or an edge

We call these operations "events," and call nodes and edges "objects."

\subsection{Recording Ideas Using Layered History Structure}

IdeaCrepe has a large advantage in that it saves all previous information which we can see by moving, deleting, or changing labels. To create those operations, we described events of IdeaCrepe in an original XML document format. In an XML document, created elements (events) are not changed or deleted. Instead, another new element is added, which is how events are recorded in IdeaCrepe.

For example, imagine a situation where we want to change the label of a node from "January" to "February." When we create a node, an element is added to the XML document. Then, if we change the label and overwrite it, the element is changed as shown in Fig. 2(a), and the information of past label "January" is lost. IdeaCrepe does not overwrite the information; instead, a relabel element is added. We update the data by piling a new layer that has a relabel element on top of the related layers (see Fig. 2(b)). 


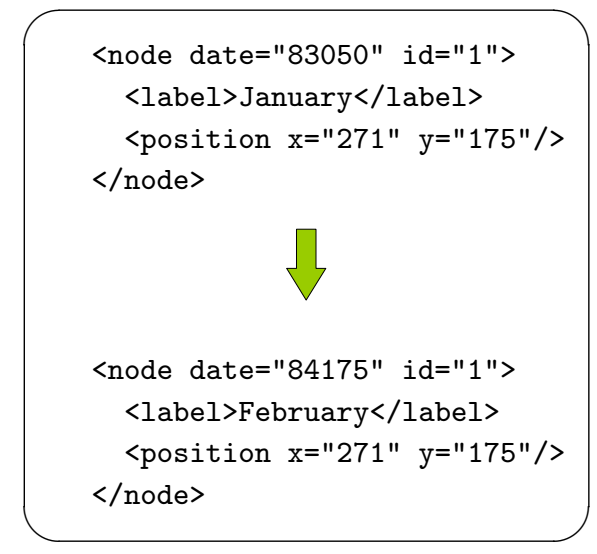

(a) By overwriting

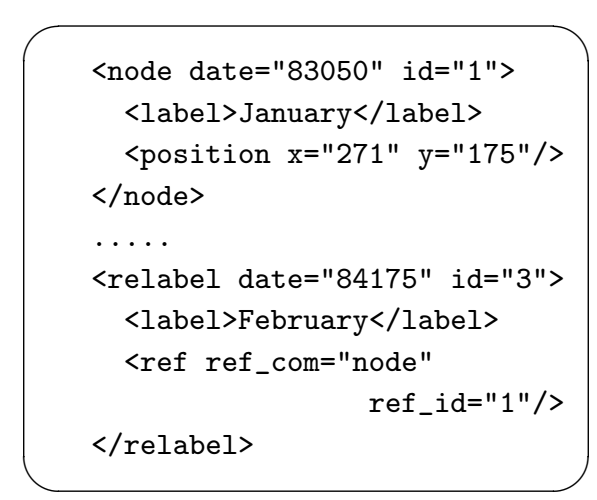

(b) Using layered history structure

Fig. 2. Save by overwriting vs. using layered history structure

\subsection{Interface of IdeaCrepe}

Screenshots of IdeaCrepe are shown in Figs. 3(a) and 3(b). IdeaCrepe has two modes. Each mode implements a different interface. Fig. 3(a) is a screenshot of the idea processor mode. Operating buttons are on the left side of the window and a canvas on the right side acts as an interface of this mode. Users do creative activities by arranging objects on the canvas. Basic functions such as file operation, and mode changing are called up easily by clicking the operating buttons.

Fig. 3(b) is a screenshot of the history layer visualization mode of IdeaCrepe. The interface of this mode is similar to the other interface; however, the right side has three sliders. Users select a key layer which users pay attention to by using the leftmost slider. The other two sliders select the range of visible layers (top and bottom).

For example, when users set all sliders at the oldest layer, only the oldest layer is visible. From this state, if users move the slider up, which indicates the top layer, they can look back at the changes in creative activities along the time series. When users want to check activities that occurred at a certain time, they only have to set the bottom slider at the beginning point, and the top slider at the ending point. Selecting the visible time period by operating the sliders enables users to look back at the evolution of their creative activities.

\subsection{User Operations}

We suppose that a mouse and a keyboard are used together to operate IdeaCrepe. Users of IdeaCrepe handle ideas by combining the following operations. 


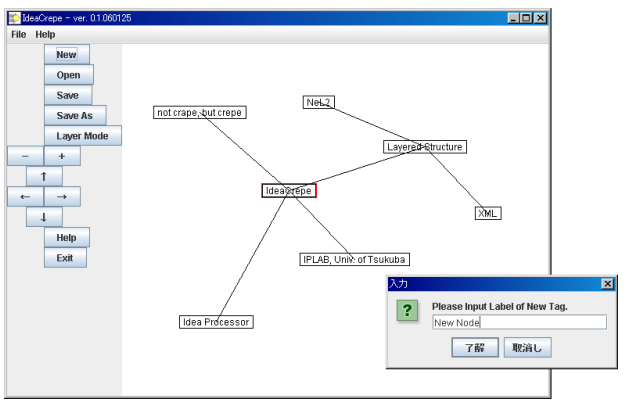

(a) Idea processor mode

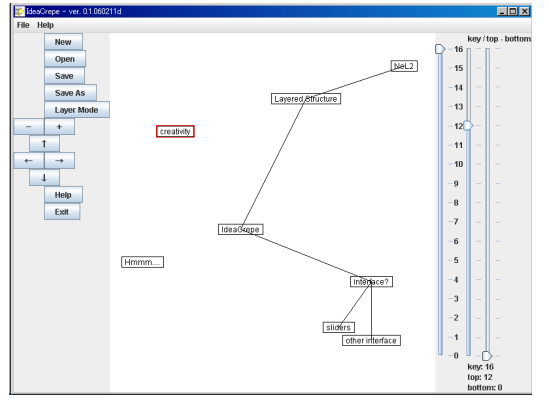

(b) Layer visualization mode

Fig. 3. Screenshots of IdeaCrepe

Create Node When a user comes up with an idea, he or she clicks any point on the canvas. A dialog box will appear, and the user can create a new node to record an idea by inputting a label in the dialog box. This node is created at the position clicked on the canvas.

Move Node and Create Edge In creative activities using pen and paper, we can pick up a piece of paper or card with our fingers and move it to a new location. When we want to create a relationship between two cards, we can draw a line between them with a pen. Those activities are simlar to moving a node and creating an edge, both of which can be done using a mouse on a computer - dragging. The difference between those operations is whether there is already another card at the dragged position, as in Fig. 4(a), or not, as in Fig. 4(b).

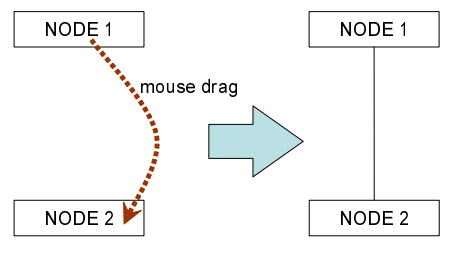

(a) Create edge

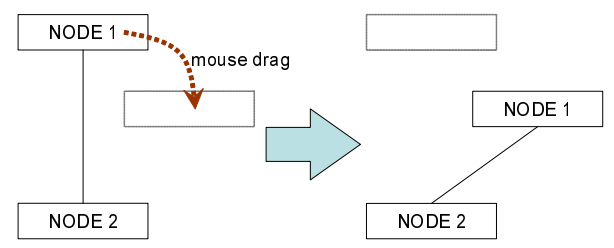

(b) Move node

Fig. 4. Move node and create edge by dragging mouse 
Change Label and Delete Objects When you want to change a label, you have to select an object to be edited. With IdeaCrepe, you can edit the label by double-clicking the node or the edge. To delete operation, users select an object to be deleted. However, users must be aware which operation is occurring: creating or deleting. Therefore, to avoid confusion, we designed the delete operation of IdeaCrepe to operate with a right click (not a left click).

Switching to History Layer Visualization Mode IdeaCrepe consists of three modules as shown in Fig. 5(a): idea processor module on which we operate knowledge creativity, file I/O module, and the history layer visualization module. By clicking the Layer Mode button on the left side of the window, IdeaCrepe switches to the history layer visualization mode (see Fig. 3(b)). Users may want as many operations in this mode as in the idea processor mode. Therefore, we implemented a way for users to change views clearly. The history layer visualization mode changes the layers using sliders.

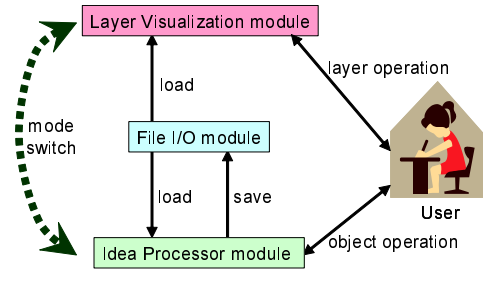

(a) Diagram of system

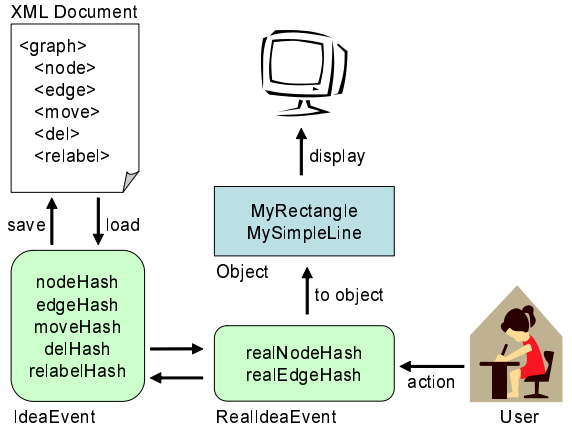

(b) Flow of events on IdeaCrepe

Fig. 5. System images of IdeaCrepe

\subsection{Visualize Ideas Using Layered Structure}

By saving ideas in a XML document using a layered structure, we can modify the ideas in the previously saved data. However, an object will be overwritten by multiple events because this tool adds new events instead of changing existing ones. To avoid this, IdeaCrepe handles data in the way shown in Fig. 5(b).

After loading an XML document, IdeaCrepe records data in an instance of IdeaEvent class and stores it in a hash table. Events with move, delete, and change labels are stored among events as overwrite nodes or edges that correspond to and can be stored in a hash table. By converting data that is stored in this hash table to the Graphics objects of Java, the data is visualized. 


\section{$5 \quad$ Evaluation and Study}

\subsection{Testing Impressions of IdeaCrepe}

We performed a creative activity by using IdeaCrepe, and tested how using this tool feels. There is still no strong evidence what effects are produced by looking back at the history of our creative activities. However, a test user said that looking back at past activities assisted him in guided future creative processes. In our future work, we should observe the circumstances of activities quantitatively, such as the number of layers and objects created, and evaluate how effective looking back at the history is.

\subsection{How to Improve Layered History Structure}

In the present implementation of IdeaCrepe, sliders operation only express layered structure. For that reason, Users have difficulty relating sliders to the concept of layers. We must add something that can remind users of the layers at a glance. Alternatively, we can replace sliders with another operation interface. For example, if users could operate the concept image of layers directly, we think that they could understand and operate the layers intuitively.

Users who look back at a process may want to continue a new branch of creativity from a certain point in the past. The piling of layers has a tree structure, so we should examine a suitable way to look at and operate such layers.

\subsection{Study of Input Devices}

IdeaCrepe was designed with the assumption that users would use a mouse and keyboard for input. However, some users operate their equipment with pens (styluses) on real world-oriented designs. Therefore, some researchers have tried to make use of merit of handwriting in creativity support [6]. Many designers like to design freely using pen and paper during the early stage of creativity [3]. We think that IdeaCrepe will have to support handwriting input using a pen as well as a mouse and keyboard. Moreover, an interface with a three-dimensional drawing board [4] may make the piling of layers more realistic.

\section{Existing Tools and Related Work}

The field of creativity support has a long history. Stefik et al. researched computer support by computer in collaboration with some people [8].

Many researchers have been developing creativity support tools. Misue et al. developed an interactive support system called D-ABDUCTOR which grasps the processes of the KJ method [9] as part of graphical thought extension [5].

Some concept mapping tools [1] have a function to playback processes, such as the tool created by Funaoi et al. [2]. This playback function is, however, uses snapshots. This function can only play back selected snapshots along the time 
series. By comparison, IdeaCrepe can show users previous states of ideas and enable them to see the changes (difference). Users are able to look back at the evolution of their creative activities with greater flexibility and variety than is possible with other existing tools.

\section{Conclusions}

We applied a layered structured network to our creativity support tool and created IdeaCrepe. This is an all-purpose tool that can operate various kinds of creative activities and that can save the histories of events in a layered structure. We can recall processes of past creative processes by looking at the history layers.

In our future work, we will modify the view of the layers to create a network diagram that users can operate layers intuitively and directly. And also, we will introduce a new operating system to replace the sliders. In addition, IdeaCrepe will draw out the creative activities of users naturally by supporting their input intuitively.

There is still no strong evidence about what effects can be produced by looking back at the history of our knowledge creative processes. In future work, we have to evaluate quantitatively how effective looking back is.

\section{References}

1. Hanson, E.: A Survey of Concept Mapping Tools. http://datalab.cs.pdx.edu/sidework/pub/survey.of . concept.maps/

2. Funaoi, H., Yamaguchi, E., Inagaki, S.: Collaborative Concept Mapping Software to Reconstruct Learning Processes. in Proceedings of the International Conference on Computers in Education (ICCE'02) (2002) 306-310

3. Landay, J. A., Myers, B. A.: Sketching Interfaces: Toward More Human Interface Design. in IEEE Computer, Vol.34, No.3 (2001) 56-64

4. Lapides, P., Sharlin, E., Sousa, M. C., Streit, L.: The 3D Tractus: A ThreeDimensional Drawing Board. in First IEEE International Workshop on Horizontal Interactive Human-Computer Systems (TableTop2006) (2006) 169-176

5. Misue, K., Sugiyama, K.: How Does D-ABDUCTOR Support Human Thinking Processes? in Proceedings of CG International '94 - Insight through computer graphics (1994) 10-21

6. Misue, K., Tanaka, J.: A Handwriting Tool to Support Creative Activities. in Proceedings of 9th International Conference on Knowledge-Based $\mathcal{G}$ Intelligent Information \& Engineering Systems (KES2005) (2005) 423-429

7. Nakazono, N., Misue, K., Tanaka, J.: NeL2: Network Drawing Tool for Handling Layered Structured Network Diagram. in Proceedings of Asia Pacific Symposium on Information Visualization 2006 (APVIS2006) (2006) 109-115

8. Stefik, M., Foster, G., Bobrow, D. G., Kahn, K., Lanning, S., Suchman, L.: Beyond the Chalkboard: Computer Support for Collaboration and Problem Solving in Meetings. in Communications of the ACM, Vol.30, No.1 (1987) 32-47

9. Kawakita, J.:The KJ-method. Chuokoron-sha (1967) (in Japanese) 